\title{
Aurora on Jupiter: A Magnetic Connection with the Sun and the Medicean Moons
}

\author{
Supriya Chakrabarti ${ }^{1}$ and Marina Galand ${ }^{2}$ \\ ${ }^{1}$ Center for Space Physics, Boston University \\ 725 Commonwealth Avenue, Boston, MA 02215, USA \\ email: supc@bu.edu \\ ${ }^{2}$ Dept. of Physics, Imperial College London, \\ Prince Consort Road, London, SW7 2AZ, United Kingdom \\ email: m.galand@imperial.ac.uk
}

\begin{abstract}
Observational astronomy began in Padova four hunderd years ago, when Galileo Galilei pointed a newly invented instrument towards Jupiter. After only one week of observations he discovered four moons circling Jupiter. In the intervening four centuries, technical progress in instrumentation and novel observational approaches have revealed much about the connection between these Medicean moons with Jupiter, none more revealing than the auroral emissions. In this paper we review observations of ultraviolet aurora made by earth-orbitting spacecraft as well as those that flew by the Jovian system.
\end{abstract}

Keywords. Medicean Moons, Aurora, Ultraviolet.

\section{Introduction}

Four hundred years ago today, observations of the night sky from this historic town revolutionized science. On this day, Galileo Galilee built his version of a toy invented by opticians, pointed it at the heavens and discovered four previously unknown moons orbiting Jupiter. This was truly a triumph of technology as these objects lied beyond the reach of the best observational tools available at the best observatories of the time. In an early demonstration of faster, better, cheaper, a scientific instrument that was shorter than a typical walking stick and took only weeks from the decision to develop to science results, established its superiority over naked-eye, castle-like observatories, that was the custom of the day. Since that auspicious day, creative minds have developed various telescopes and tools that have revealed many unexpected phenomena and surprising characteristics including those that are truly invisible to the human eye.

Four centuries of advances in technology have allowed us to answer many of the original questions on these Medicean moons. Our instruments on remote mountain tops, on space platforms near Earth, as well as those that have orbited Jupiter and flew past the Jovian system have given us a closer look of these bright points of lights. We now have a fairly good understanding of the major physical attributes of these moons, know how different they are from our own and from each other and why.

In this paper we will review observational progress made in our understanding of Jupiter and the Galilean moons and their magnetic connections as revealed through aurora. Auroral emissions and their morphology contain information on atmospheric composition, plasma interactions, magnetic field configuration, and magnetosphere-ionosphere coupling processes. Jupiter and its moons provide us with a rich wall-less laboratory environment that is accessible to probing by our spaceborne and ground-based observatories.

This is a chronicle of the triumph of technology. In the past two decades, new instruments behind large telescope have obtained conclusive evidence of planets around distant 
stars. We can now set our sights on these planets and, if the technical feats continue at their past or current pace, it would not be unexpected for another toolmaker to discover moons orbiting around one of these planets within this century.

\section{Aurora: Background}

On Earth, brilliant and dynamic auroral displays have conjured many colorful folklores and have been the subject of fascinating mythology (Eather (1980)). High occurrence frequency in certain geographic locations (now called auroral zones) and east-west extension of auroral displays which are correlated to large magnetic fluctuations established their geomagnetic connection. Even though people have studied aurora as long as we have studied the sky, there have been no less than three relatively recent instances when it was necessary to define aurora (Galand \& Chakrabarti (2002), Clarke (2004) and Bharadwaj (2005)).

Pierre gassendi of the Transit of Mercury fame, coined the term Aurora Borealis (Northern Dawn) in 1621. Aurora observed at Earth was first defined by its temporal, spatial, spectral, and magnitude characteristics. However, these characteristics vary greatly from one Solar System body to another, as they depend on atmospheric composition, magnetic field configuration, energetic particle origin and acceleration mechanisms, and plasma interaction involved in their production. As a result, a more general definition based on their origin is needed for defining what aurora is beyond the Earth. Galand and Chakrabarti (2002) were the first to propose a definition applicable to bodies in the Solar System, followed later by Clarke (2004) and Bhardwaj (2005). A definition allows to make a classification, yielding a deeper understanding of plasma interactions in the Solar System.

Galand \& Chakrabarti (2002) defined aurora to be optical manifestation of the interaction of extra-atmospheric energetic electrons, ions, and neutrals with an atmosphere. Clarke (2004) defined aurora to be atmospheric emissions produced by the impact of external energetic particles - a definition similar to that of Galand \& Chakrabarti (2002) However, he was conflicted on the issue of emission produced by energetic neutrals, which do not follow magnetic field lines. He noted that while this (the requirement of a magnetic field or localized region of particle precipitation) has been part of the historic definition of the aurora, it would require altering the literature as it has naturally evolved. Bharadwaj (2005) required the presence of a magnetic field for optical emissions to be classified an aurora and accepted that such emissions are generally localized in the high latitude (presumably magnetic latitude) regions. He noted, whether the magnetic field is intrinsic (natural) or induced, or of a nearby magnetic body (i.e., of planets for planetary satellites), or the interplanetary magnetic field, is a separate question. He added, even in cases of neutrals causing an aurora at low latitudes, they have been under the influence of a magnetic field until they got converted to neutral in the charge-exchange reaction. Fortunately, for the cases of auroral emissions on Jupiter and the Medidean moons, the thread that connects them is the magnetic field and the emissions are produced by energetic ions or electrons, thus, avoiding any confusion.

While the physical and chemical processes that result in optical emissions in aurora are governed by the same underlying physics, the spatial, temporal and magnitude scales, and energetics vary enormously because the atmospheric composition, energy source and magnetic field geometry are often unique to each type of object. Thanks to such rich and diverse auroral processes, a comparative approach can be applied to develop a comprehensive understanding of one of the complex interactions in the solar system. 


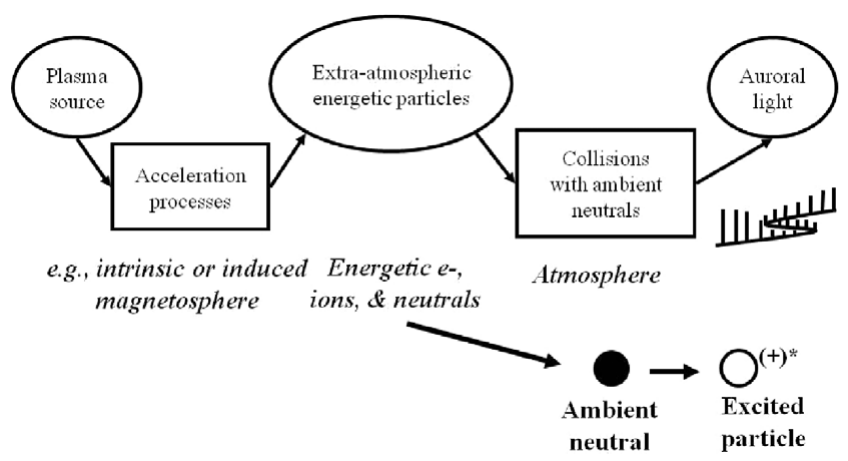

Figure 1. A cartoon depiction of processes leading to auroral emissions.

The general picture that is consistent with all these descriptions has been summarized in Fig. 1.

In this paper we will review the magnetic connections between Jupiter and the Medicean moons as manifested through ultraviolet aurora. While auroral observations have been made from IR to X-rays and beyond, we chose to restrict the discussions to UV emissions only. The UV emissions allow examination of spectral lines of many of the common atmospheric species in a compact wavelength region. Furthermore, the excitation mechanisms are simple and since the energy threshold are high $(>10 \mathrm{ev})$, complex photochemistry and albedo from lower atmospher, which complicates interpretation of visible emissions, can be ignored. However, the observed UV emissions are sometimes altered by radiative transfer processes, which need to be carefully accounted in the models.

\section{Observational and Instrumentation Issues}

The progress in optics, detector and space technology made our understanding of the complex processes that govern plasma interactions along magnetic fields leading to auroral emissions, which are tracers of solar wind-magnetosphere-ionosphere-atmosphere coupling. The Earth's aurora, being the most accessible of solar system objects, has been studied extensively. Terrestrial aurora has been scientifically studied since the 18th Century (see for example, Mairan (1733) and other listed in Chamberlain (1995)). Key observational milestones include the first spectroscopic study (Angstrom (1869)) and the first all-sky auroral morphology study (Gartlein (1947)). Since then, every advance in optics, detector and related technology, just like astronomical studies after Galileo, have been implemented in auroral observations.

Ground-based photometric, spectroscopic and imaging observations have established auroral spectra in the visible portion of the electromagnetic spectrum and their morphology over a given site (Vallance Jones [1974). Until recently, ground based observations have been limited to night time conditions centered around new moon. Recent advances in optical technology have demonstrated that selected auroral emissions could be observed round-the-clock even under fully sunlit conditions (Chakrabarti \& Pallamraju (2006)).

With the availability of array detectors and space platforms, our understanding of the aurora increased dramatically. Ultraviolet observations, possible only from space-based 
platforms, have opened up the ability to observe sunlit aurora. Early rocket and satellite based spectroscopic observations (Crosswhite et al., (1962); Sharp (1971); Eastes \& Sharp (1987); Huffman et al., (1980); Paresce et al., (1983a); Paresce et al. (1983b); Chakrabarti et al., (1985); Chakrabarti et al., (1986) and references therein) have shown terrestrial auroral spectrum is dominated by $\mathrm{O}^{+}$emissions shortward of $90 \mathrm{~nm}$ while the spectral region between 90 and $200 \mathrm{~nm}$ contain many emission features of $\mathrm{H}, \mathrm{O}, \mathrm{N}, \mathrm{O}^{+}, \mathrm{N}^{+}$, $\mathrm{H}_{2}$, and $\mathrm{N}_{2}$. Spectroscopic studies of UV aurora have used emission intensity ratios to characterize the particle energies in the sunlit cusp, polar cap and nightside aurora (e.g., Chakrabarti et al., (1986)). Global imaging in the visible (e.g., DMSP), ultraviolet (e.g., Kyokko, Dynamics Explorer, Polar Bear, Viking and Polar/UVI, IMAGE) and X-rays (e.g., Polar/PIXIE) provided a better understanding of auroral processes. The size of the auroral oval derived from global images has been related to solar wind parameters (Siscoe (1991)). Evolution of the global aurora at up to $50 \mathrm{~km}$ spatial and 1 minute temporal resolutions have been examined to understand the substorm onset and poleward expansion [e.g., Craven \& Frank (1987) and Shepherd et al., (1987)].

Satellite borne ultraviolet spectrographs using microchannel plates, a key component of the night vision technology (Bowyer et al., (1981)), established the geographic morphology and relationship with geomagnetic and solar activities for terrestrial aurora (Paresce et al., (1983a); Paresce et al. (1983b)). Microchannel plates allowed observations of very low light levels as it enabled photon counting - building images or spectra one photon at a time. An imaging UV spectrograph aboard the International Ultraviolet Explorer (IUE) observed auroral emissions from Jupiter in the $120-170 \mathrm{~nm}$ band (Clarke et al., (1980)), which was quickly followed by Voyager 1 observations in the $60-160 \mathrm{~nm}$ band (Broadfoot et al., (1981)). Voyager observations of Jupiter's UV aurora were found to follow closely to the magnetic field lines connected to the Io Torus.

Charged Couple Devices (CCDs) and Intensified Charge Coupled Devices (ICCDs) have been used in auroral imaging and spectroscopy applications. With the spectra established, spectacular images and indeed movies of Jovian aurora in selected UV wavelength bands have been obtained by the Hubble Space Telescope (HST) which vividly showed the magnetic connections with the moon (Fig. 2).

Similar to Jupiter, the Medicean Moons have atmospheres. They are also embedded in Jupiter's magnetic environment where energetic particles are present, primarily from the Jovian system and with a small contribution from the Sun. As a result, we expect to see aurora, the optical manifestation resulting from complex and dynamic interactions between them, on each of these objects. The composition of the atmospheres of Jupiter and its Medicean moons, their magnetic field strength and geometry are very different from that of the Earth and from each other, which results in vastly different auroral signatures (see Bharadwaj \& Gladstone (2000) for a comprehensive survey of auroral processes on Jovian planets). Through observations of UV aurora and comparing their brightness characteristics as well as spatial and temporal morphology against those observed in X-rays and IR, one can infer atmospheric composition and distributions, energy of the particles and trace the magnetic field configuration and plasma interactions.

\section{Determination of Physical Properties and Processes from Auroral Emissions}

Several techniques have been employed in inferring emission sources, key physical processes in the aurora and the environment they are produced; two common ones are pictorially depicted in Fig. 3 Fox et al., 2008. 


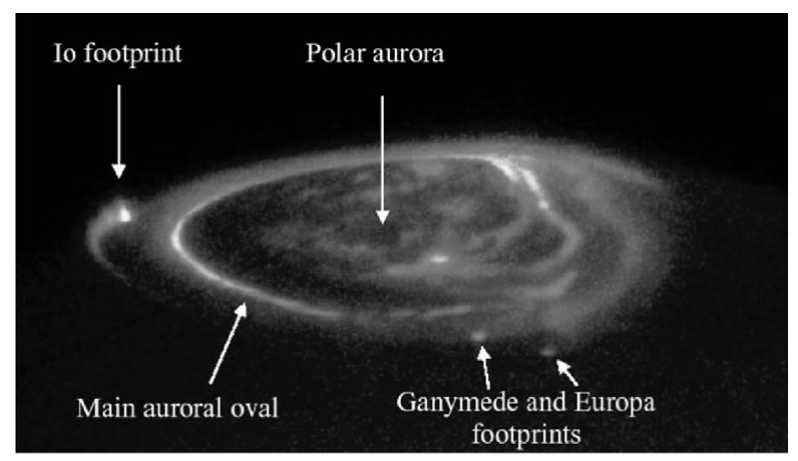

Figure 2. HST observations of UV aurora (false color) on Jupiter showing the presence of a main oval and polar cap aurora, similar to Earth (though at Jupiter, the main oval is associated with the breaking from co-rotation, unlike at Earth). The polar cap aurora was very dynamic and influenced by solar wind conditions. In addition, unlike at Earth, Jovian aurora also shows features magnetically connected to its moons. They represent the magnetic footprints of the moons in the jovian atmosphere. Note that Callisto footprint cannot be seen as its location is predicated within the main oval. Clarke et al. (1980) Photo Credit: NASA

The first one of these techniques is based on color intensity ratio. Noting that higher energy particles penetrate deeper in an atmosphere, the intensity ratios of selected emission lines observed from a vantage point above the atmosphere have been used to infer atmospheric composition as well as characteristic energy of the incident particles (see Table 1). Similar technique has been applied for the case of the Earth's sunlit cusp, polar cap as well as nightside aurora to characterize incident particle energy by examining intensity ratios of selected emission lines (Chakrabarti (1986)).

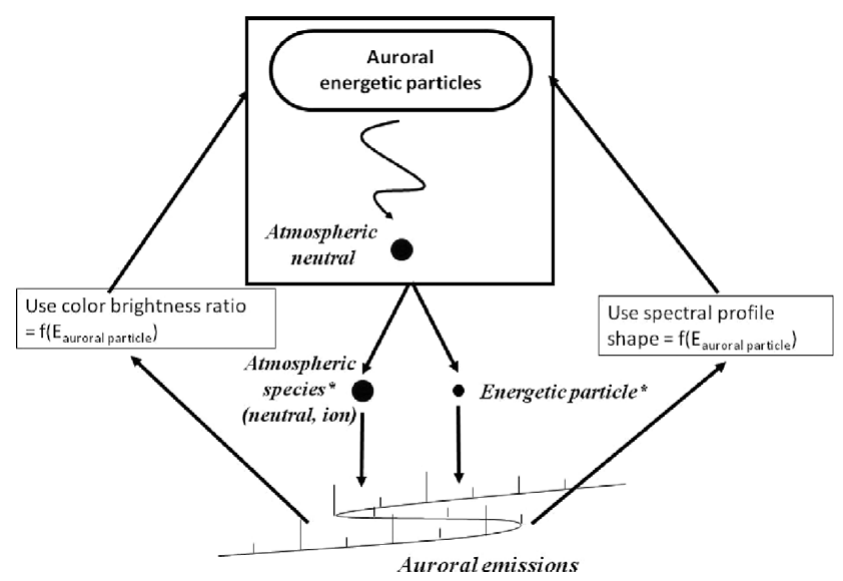

Figure 3. Two common methods of obtaining physical parameters from auroral emissions.

As an example, intensity ratios of two far ultraviolet windows covering $\mathrm{H}_{2}$ bands at 123.0-130.0 nm and 155.7-161.9 nm have been used for Jupiter. Emissions in the shorter 
Table 1. Example of Auroral Spectroscopic Analysis Using Color Ratios

\begin{tabular}{lcc}
\hline Color Ratio & Earth & Jupiter \\
\hline Two Spectral Bands Chosen in & $N_{2} \mathrm{LBH}$ & $\mathrm{H}_{2}$ Lyman and Werner bands \\
One band Strongly Abosorbed by: & $\mathrm{O}_{2}$ & $\mathrm{CH}_{4}$ \\
Electron Energy Range Covered & $0.2-20 \mathrm{KeV}$ & $10-200 \mathrm{KeV}$ \\
Type of Aurora identified: & Discrete Electron Aurora & Hard e- component of Electron Aurora \\
\hline
\end{tabular}

wavelength spectral window is strongly absorbed by methane, while emission in the longer wavelength window is absorbed only weakly. As a result, this intensity ratio is a function of the energy of incident electrons in an aurora as represented by the altitude of the methane layer on Jupiter (note, the primary absorber for terrestrial auroral emissions in the UV is $\mathrm{O}_{2}$ ). Pallier \& Prange (2004) and Rego et al., (1999) used this technique with HST/STIS data to infer the energy of the electrons in Jupiter's aurora. Similar analyses have also been applied to spectroscopic data obtained by IUE, HUT, Galileo/EUVS, Cassini/UVIS as well as by New Horizon/Alice.

The second technique is based on the spectroscopic analysis of emission line spectra observed at high spectral resolution. The shape of the same spectral line can carry the signature of different physical processes. For example, the hydrogen Lyman alpha emission line in a terrestrial aurora as seen from space was observed to be red shifted by $0.13 \mathrm{~nm}$. The spacecraft which made these observations was located at $600 \mathrm{~km}$ altitude, significantly above the peak emitting region. Galand et al., (2002) interpreted these observations as emissions produced by precipitating protons and their interactions with the atmospheric neutral species.

The line shape of the H Lyman Alpha line in Jovian aurora observed by the HST/GHRS is significantly more complicated. The emissions show a self-reversed core which is red shifted from the geocoronal rest frame. While core reversal is common is solar and stellar spectra, such a feature had not been seen in planetary atmosphere and was first discovered in Jovian aurora. Further analysis of the observed red shift and the asymmetric line profile revealed that the core reversal is located at the Jovian rest frame thereby indicating that the emission source is deep in the Jovian atmosphere, below the atomic hydrogen atmosphere, which absorbs the photons at its rest frame. Due to the relative motion of the Earth and Jupiter, the Jovian rest frame was red shifted from the geocornal rest frame (see Prange et al., (1996)). Thus while H Lyman alpha emission line shape in terrestrial aurora can be used to infer properties of incident protons on the Earth, the line shape of the same spectral line in Jovian aurora carry the signature of precipitating electrons.

\section{UV Emissions connected to the Medicean Moons and their Morphology}

The connection between auroral emissions and the magnetic field of the planet has been well established by examining the morphology of the location and geometry of terrestrial aurorae. They have also demonstrated a strong solar wind connection. In a study involving Coronal Mass Ejection (CME) from the Sun, it was shown how such an event leaves a profound signature through auroral emissions; four different spacecraft recorded auroral emissions due to a single CME event on the Earth (Polar spacecraft), Jupiter (Galileo and Cassini spacecraft) and Saturn (HST) (Prange et al., (2004)).

Fig. 2 shows an image of the FUV $(115-170 \mathrm{~nm})$ aurora recorded by HST. From these and other multiwavelength observations, it has also been established that several 
auroral processes are active on Jupiter (Vasavada et al., (1999)). The main oval observed in the UV, visible, hard X-ray, and near IR and produced by magnetospheric electrons while the soft X-ray are produced by energetic oxygen and sulfur ions. The polar cap emissions, which are very dynamic, have been seen in UV are responsive to solar wind conditions. Finally, localized emissions seen at the magnetic footprints conecting the Medicean moons and believed to be due to energetic electrons Thus, auroral emissions, at least emissions in the auroral zone, connect the Solar Wind to the magnetosphere, neutral atmosphere and ionosphere of a planet and its moon through diverse magnetic field geometry.

The auroral emissions and their temporal morphology have been studied in detail using ground- and space-based instruments. Prange et al., (1996) established that the FUV auroral "spot" due to Io on Jupiter was located in a region consistent with the IR emissions (Connerney (1993)); however, both were seen poleward of model footpoint of the magnetic field lines (Hill et al., (1983)) that connected Io. The emission brightness were found to represent $25 \%$ of the energy produced due to the motion of Io across Jovian magnetic field lines - a value that corresponds to an energy flux that equals the entire terrestrial auroral energy flux concentrated in a $10^{4} \mathrm{sq} . \mathrm{km}$ area.

The location of the magnetic footprints observed in the UV has been used to constrain the topology of the magnetic field (Grodent et al., 2008). Grodent et al. (2008) extracted the location of the magnetic footprints of the three Medicean moons, Io, Europa, and Ganymede, from individual UV images taken by HST in order to derive observed, reference contours. By comparison of these contours with the moon footprint locations extracted from the VIP4 magnetic field model (Connerney et al., 1998), they inferred the presence of a magnetic field anomaly in the northern hemisphere of Jupiter.

Recently, Wannawichian et al., (2010) have examined a decades of FUV emissions recorded by the HST at the footprint of the Medicean moons Io, Ganemede and Europa in terms of the electrodynamic interactions between Jovian magnetosphere and the atmospheres of the moons. After correcting for limb brightening effects, the authors found a factor of $8-10$ brightness variations at the footprint of Io as a function of the location of Io (System III longitude). The intensity showed a two peak variations, with the second being approximately a factor of two dimmer than the first. Both peaks are observed when Io is near the plasma equator and suggests a strong connection between the brightness and the plasma density of the torus where the moon is located, thereby indicating a strong control by mass pick up processes.

The absolute brightness of the footprint of Ganymede is approximately a factor of two dimmer than that of Io. Ganymede also shows a variation of brightness that is correlated with the System III longitude and a peak near the equator. However, due most likely to its lower brightness, similar relations could not be established for Europa. Unlike Io, plasma densities at the locations of Ganymede and Europa are significantly lower. The brightness variations at these two locations suggests a stronger solar wind connection. The Jupiter and its Medicean moons presents a wall-less laboratory where the complex plasma processes manifest in bright UV emissions in the auroral zone.

\section{Aurora on the Medicean Moons}

Two Jovian Moons, Io and Ganymede, have substantial extended atmospheres. They are also immersed in Jupiter's magnetosphere. The location of the bright footprints seen near the auroral oval of Jupiter and the temporal variation of their brightness correlate 
well with the magnetic field geometry. It is therefore reasonable to expect that just like their footprints on Jupiter, some aurora-like features should be present on these moons.

Observational evidence of UV auroral features on Io was reported by Roesler et al., (1999). Using the Space Telescope Imaging Spectrograph (STIS) the authors observed bright OI $135.6 \mathrm{~nm}$ and SI 138.9, SI 142.9 and SI $147.9 \mathrm{~nm}$ emissions near Io's equator that shifted positions with the orientation of Jupiter's magnetic field. While the authors also observed time-varying HI Lyman alpha emissions from Io's polar region, they are not believed to be due to aurora-like excitation process. More recently, Retherford et al. (2007) reported the observations of Io's aurora in eclipse by New Horizons ultraviolet spectrometer. These observations revealed the influence of plumes on Io's electrodynamic interactions with Jupiter's magnetosphere with the volcanoes supplying up to $3 \%$ of the dayside atmosphere.

After the detection of atmospheric atomic oxygen emissions by Hall et al., (1998), Feldman et al., (2000) used a similar approach on Ganymede and confirmed bright spots in OI $135.6 \mathrm{~nm}$ emissions on the trailing side near its poles (latitudes greater than $40^{\circ}$ ). These STIS observations showed temporal variations as well as inter hemispheric variations, which the authors attributed to aurora-like excitation mechanism. The location of auroral emissions near the poles is explained by the presence of an intrinsic magnetic field at Ganymede discovered by the Galileo spacecraft [Kivelson et al. (1996)]. Recent 3D multi-fluid simulations have confirmed the auroral nature of these emissions Paty \& Winglee (2004).

\section{Concluding Remarks}

Ultraviolet aurora on Jupiter has revealed the complex magnetic connection with the galilean moons. The moons have left bright ultraviolet footprints on Jupiter. The magnetic field lines on the moons also glow in UV light. These emissions and their spatial and temporal morphology have been used to reveal the physical processes involving magnetospheric plasma and neutral atmospheric species.

The next 400 years will see a steady progress in optical technology. Already interferometric tools are being developed (See for example, Hicks et al. 2009 and references therein), that will be capable of directly imaging Jupiter-like planets around Sun-like stars within a decade. It should not strain anyone's imagination to envision a time within the next century when another Galileo will point an instrument towards such an exoplanet and find their own Medicean moons.

\section{Acknowledgements}

The work at Boston University was supported in part by NASA grants NNX06AC04G and NNG05WC17G.

\section{References}

Angstrom, A. J., 1869, Spectrum des Nordlichts, Pogg. Ann, 137, 161

Bharadwaj, A., 2005, EOS Trans. AGU, 86(11), doi:10.1029/2005EO110007

Bharadwaj, A. \& Gladstone, G. R., 2000, Rev. Geophys., 38, 285

Bowyer, S., Kimble, R., Paresce, F., Lamptom, M., \& Penegor, G., 1981, Appl. Opt., 20, 477

Broadfoor, A. L., Sandel, B. R., Shemansky, D. E., McConnell, J. C., Smith, G. R., Holberg, J. B, Atreya, S. K., Donahue, T. M., Strobel, D. F., \& Bertaux, J. L. 1981, J. Geophys. Res., 86, 8259, doi:10.1029/JA086iA10p08259

Chakrabarti, S., 1985, J. Geophys. Res., 90, 4421

Chakrabarti, S., 1986, J. Geophys. Res., 91, 8065 
Chakrabarti, S. \& Pallamraju, D., 2006, in: Marc Duldig. (Ed.) Advances in Geosciences, Volume 2: Solar Terrestrial (ST),World Scientific Co., Pte. Ltd., Singapore, p. 201

Chamberlain, J. W., 1995 Physics of Aurora and Airglow, (American Geophysical Union, Washington, D. C.),

Clarke, J. 2004, EOS Trans. AGU, 85(52), 567

Clarke, J., Moos, H., Atryea, S., \& Lane, A. L., 2004, Astrophys. J., 241, L179, doi:10.1086/183386

Connerney, J. E. P., baron, R., Satoh, T., \& Owen, T. 1993, Planetary Science, 262, p. 1035.

Craven \& Frank, 1987 J. Geophys. Res., 92, 4565

Crosswhite, H., Zipf, E., \& Fastie, W., 1962, Opt. Soc. Am., 52, 643

Eastes, R. \& Sharp, W., 1987, J. Geophys. Res., 92, 10095

Eather, R. H., 1980, Majestic Lights, American Geophysical Union, Washington, D.C.

Feldman, P., McGrath, M., Strobel, D. Moos, H., Retherford, K., \& Wolven, B. 2000, Astrophysical L., 535, 1085

Fox., J. L., Galand, M. I., \& Johnson, R. E. 2008, Space Sci. Rev., 139, 3-62, doi:DOI $10.1007 / \mathrm{s} 11214-008-9403-7$

Galand, M. \& Chakrabarti, S. 1998, in: M. Mendillo, A. Nagy, \& J. H. Waite (eds.), Atmospheres in the Solar System, (Washington, D. C., AGU Monograph), 130, p. 55.

Galand, M., Lummerzheim, D., Stephan, A., Bush, B., \& Chakrabarti, S. 2002, J. Geophys. Res., 107(A7), 10.1029/2001JA000235

Gartlein, C. W., 1947, National Geographic Magazine, 673, Nov.

Hall, D., Feldman, P., McGrath, M., \& Strobel, D. 1998, Astrophys. J., 499, 475

Hicks, B., Cook, T., Lane, B., \& Chakrabarti, S. "Monolithic achromatic nulling interference coronagraph: design and performance", Appl. Opt., 48, 4963-4977, 2009.

Hill, T. W., Dessler, A. J., \& Goertz, C. K. 1983, in: A. J. Dessler, ed., Physics of the Jovian Magnetospheres, (Cambridge Univ. Press), p. 353.

Huffman, R. E., Leblanc, F. J., Larrabee, J. C., \& Paulsen, D. E., 1980 J. Geophys. Res., 85, 2201, doi:10.1029/JA085iA05p02201

Kivelson, M. G., Khurana, K. K., Russell, C. T., Walker, R. J., Warnecke, J., Coroniti, F. V., Polanskey, C., Southwood, D. J., \& Schubert, G., 1996 Nature 384, 537

Mairan, J. J. D. de, 1733 Imprimerie Royale, Paris

Pallamraju, D. \& Chakrabarti, S., 2005 Geophys. Res. Lett., 32, L03S10,doi:10.1029/2004GL021417

Pallier, L. \& Prangé, R., 2004 Geophys. Res. Lett., 6701, doi:10.1029/2003GL018041

Paresce, F., Chakrabarti, S., Bowyer S., \& Kimble, R., 1983a J. Geophys. Res., 88, 4905

Paresce, F., Chakrabarti, S., Kimble, R., \& Bowyer S., 1983b J. Geophys. Res., 88, 10247

Paty C. \& Winglee, R. 2004 Geophys. Res. Lett., 31, L24806, doi:10.1029/2004GL021220

Prangé, R., Rego, D., Southwood, D., Zarka, P., Miller, S., \& Ip, W., 1996 Nature, 379, 323

Prangé, R., Pallier, L., Hansen, K. C., Howard, R. Vourlidas, A. Courtin, R., \& Parkinson, C., 2004 Nature, 432, 78

Rego, D., Prangé, R., \& Ben Jaffel, L., 1999 J. Geophys. Res.,104, 5939

Retherford, K. D. \& 20 co authors, 2007 Science, 318, 237

Roesler, F., Moos, H. W., Oliverson, R., Woodward, Jr., R., Retherford, K., Schreb, F., McGrath, M., Smyth, W., Feldman, P., \& Strobel, D., 1999,Science, 283, 353

Sharp, W., 1971 J. Geophys. Res., 76, 987

Shepherd et al., 1987 Geophys. Res. Lett., 14, 395,doi:10.1029/GL014i004p00395

Siscoe, G., 1991, in, C.-I. Meng, M. J. Rycroft, \& L. A. Frank eds. Auroral Physics, 159

Vallance Jones, A., 1974 Aurora, D. Reidl Publishing Co., Dordrecht Boston

Vasavada, A. R., Bouchez, A. H., Ingersoll, A. P., Little, B., Anger, C. D., \& the Galileo SSI Team, 1999 J. Geophys. Res., 104, 27133, doi:10.1029/1999JE001055

Wannawichian, S., Clarke, J. T., \& Nichols, J. D., 2010 J. Geophys. Res., 115, 2206, doi:10.1029/2009JA014456 